\title{
Equipoise as a means of managing uncertainty: personal, communal and proxy
}

\author{
Priscilla Alderson Institute of Education, University of London
}

\begin{abstract}
Equipoise is advocated as a means of achieving high scientific and ethical standards in randomised trials. As used in the context of research the word describes a state of uncertainty characterised by the belief that in a trial no arm is known to offer greater harm or benefit than any other arm. Clinicians who lack personal equipoise are advised to accept clinical or communal equipoise, based on current unresolved disagreement among the medical profession. Equipoise is mainly discussed in the literature as an issue for senior doctors and research directors. Limitations of professional equipoise are reviewed, and data on the neglected topic of patients' equipoise are reported using the example of breast cancer trials. In theory, a patient who gives informed and voluntary consent to enter a randomised trial has achieved the equilibrium of equipoise. In practice, equipoise among patients ranges from personal to proxy acceptance.
\end{abstract}

\section{Introduction}

Equipoise is advocated as a means of achieving high scientific and ethical standards in randomised trials. A state of uncertainty, it is characterised by the belief that, in a trial, no arm is known to offer greater harm or benefit than any other arm. This belief enables clinicians sincerely to recommend to their patients that they enter randomised trials. ${ }^{1}$ Clinicians who lack personal equipoise are advised to accept a communal position, relying on collective or clinical equipoise based on current unresolved disagreement or uncertainty among the medical profession. ${ }^{2}$ It has been said that there must be collective uncertainty about optimal choices, or communal equipoise, in order for a trial to be ethical. Otherwise there is a danger that the trial will not be properly aimed at maximising benefits to future patients. ${ }^{3}$ Despite claims that patients in trials tend to have better outcomes, so far there is little clear evidence for this. ${ }^{4}$

\section{Key words}

Research ethics; equipoise; informed consent; randomised trials.
Equipoise is mainly seen and discussed as an issue for senior doctors, who may have to circumvent the effects on trials of bias among nurses and junior doctors. Silverman, for example, describes how junior doctors and nurses in his neonatal unit would select the coloured ball linked to their preferred treatment for each baby, instead of picking one at random. When he wrote the treatment arms on slips of paper and put each one in an envelope, the staff would hold up the envelopes to the light, in order to select the treatment by choice instead of by chance. Silverman described how he then made the envelopes opaque. ${ }^{5} \mathrm{He}$ did not appear to consider the ethical dilemmas for nursing and junior medical staff who do not have equipoise, and yet are expected to enrol babies in trials, inform and consult with parents, and act as the main link between research, treatment and care of the family.

The concept of communal equipoise has been critically analysed, to support the conclusion that it cannot "do what it was hoped it could". ${ }^{6}$ The author, Gifford, asks, what exactly is the "medical community"? How many of its members have to be uncertain, and how uncertain do they have to be, before communal equipoise can reasonably be claimed? Since complete unanimity is seldom achieved, can communal equipoise almost always be claimed? How long can uncertainty reasonably be claimed to justify the later stages of a long trial? Does the uncertainty of a few specialists count for more than the "not-irrational hunch" shared by many doctors concerning certain treatments? Doctors are guided by experience, observation, tradition and other potentially valuable sources of knowledge besides randomised research; the nature and sources of communal medical knowledge, and therefore the grounds of medical ignorance, have not been clearly agreed. Advocates of trials claim that $85 \%$ of medical interventions are so far unevaluated, though evidence for this claim is uncertain. ${ }^{7}$ They also assert that the controlled experiment of a trial is more ethical than the "uncontrolled experiment" of routine but unevaluated interventions. Yet this claim plays on a double meaning of "uncontrolled". Treatment which has not been subject to a controlled trial is "uncontrolled" in a strictly scientific sense, but is not 
necessarily "uncontrolled" in the everyday meaning of chaotic, irresponsible, casual and even callous, which the word can imply.

The literature on equipoise seldom mentions the patients' views. In theory, a patient who consents to enter a randomised trial has achieved equilibrium, and accepts the known risks and benefits of undertaking or of foregoing each treatment arm. Equipoise, in the literature, is usually implicitly confined to ignorance about the relative efficacy of the outcome of each treatment arm. Yet the patient's understanding of equipoise has to go beyond outcome to include process. For example, a woman agreeing to enter the trial of adjuvant treatment for ductal carcinoma in situ (DCIS) would need to go beyond the scientific and philosophical, abstract meaning of equipoise. She would have to consider the process, the likely experience of going through each of the treatment arms, their effects on her life and body and on people who depend on her, and the possible short and long term effects. In many trials, similar treatments are compared for relatively low risk disease. In some breast cancer trials, patients are randomised to very dissimilar treatment arms, at a time when they are shocked by the diagnosis, and fearful of an unexpectedly early death and of mutilation and distressing treatments. Many women are amazed when they are informed about the extent of medical uncertainty and the concepts of randomised trials and equipoise. For them to understand, evaluate and come to terms with the information and to arrive at, or to achieve, an intellectual and emotional state of equipoise can be a long hard struggle.

In theory, each woman would have to reach some kind of equilibrium about each of the options, although in some versions of the DCIS trial the arms range after surgery from annual observations only, to having tamoxifen, radiotherapy and chemotherapy. Can patients achieve equipoise? Do they wish to go through the intellectual and emotional process of accepting the uncertainties and risks that equipoise and randomisation can entail? This paper reports part of a study of professional and public views on equipoise in breast cancer trials. ${ }^{8}$

\section{Professional and public views on equipoise}

In a multi-centre pilot study in England, during 1993 we interviewed 50 consecutive patients aged 33 to 83 after treatment for primary breast cancer (referred to as the treated women), and 40 doctors, nurses and radiographers aged 29 to 58 , working in breast cancer in specialist centres or in general surgery units; 93 women, aged 50 to 73 , replied to our questionnaire survey sent to 161 consecutive names on breast screening lists (referred to as the screened women). A few of the treated women said they had taken part in clinical breast cancer research, some said they thought they might have been $\stackrel{\text { Tे }}{\overrightarrow{0}}$. involved, but they were unsure; with others, we? thought from their replies and knowledge of the centre where they were treated that they might have been in trials but we were uncertain.

Our research raised ethical problems. We were $\frac{\bar{\sigma}}{\bar{c}}$ asked by research ethics committees not to approach $\overrightarrow{\widetilde{\sigma}}$ women until three months after their diagnosis of $\varrho$ primary breast cancer, so we were relying on theiro memories of complex and distressing experiences. $\vec{\circ}$ As breast cancer is a chronic disease - some women were still having treatment and others would eventu- $\vec{\omega}$ ally need further treatment - we had to be very careful to try not to affect their confidence in the professionals treating them. If an interviewee gave over-optimistic responses about rates of mortality or $\omega$ the success of treatments we did not inform her $\vec{\omega}$ about the actual rates, although we sent a short ${ }^{\circ}$ report to every respondent when the pilot stage was 음 completed, with details about how they could obtain further information if they wished to. To respect $\subseteq$ confidentiality and privacy we did not see the $\widetilde{\sigma}$ patients' notes or discuss individuals with the $\operatorname{staff}_{\overrightarrow{0}}$ caring for them, which is why the numbers enrolled $ळ$ in research were uncertain. To check patients' notes for records on involvement in research is a compli-o cated exercise; a study of 334 women treated for invasive breast cancer in south-east England found that only 17 case notes recorded that the woman $\frac{}{\mathbb{Q}}$ took part in a clinical trial, 0.05 per cent. ${ }^{9}$

Few of the health professionals had heard of the $\overline{\bar{a}}$ word "equipoise", though most accepted the concept. Table 1 shows that only a quarter of the

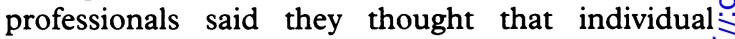
doctors could achieve equipoise, still fewer said that the whole breast care team working on a trial could $\frac{\Phi}{\sigma}$ share equipoise, or that some patients could achieve 3 . it. One nursing sister compared receiving a diagnosis of breast cancer to being on a ship with a tilting deck 3 when everything is sliding into the sea. ${ }^{10}$ She thought 0 that to such patients, at such a time, learning about randomisation and the uncertainty concerningo optimal treatment could seem like having the last hope removed. "Complex" replies from all groups $\widetilde{N}$ included discussion of the degrees of intellectual N conviction and emotional commitment required, the N types of people or research interventions concerned, 0

Table 1 Views of 40 breast cancer professionals on whether equipoise is possible for individual doctors, for the whole breast care team, or for certain patients.

\begin{tabular}{|c|c|c|c|}
\hline$\%$ Replies & Doctors & Breast care team & Patients \\
\hline Yes & 25 & 13 & 18 \\
\hline No & 35 & 58 & 38 \\
\hline Complex & 20 & 10 & 18 \\
\hline Don't know & 13 & 8 & 10 \\
\hline No reply & 8 & 13 & 18 \\
\hline
\end{tabular}

Note: Responses have been recorded as percentages to allow easier comparison; they are rounded up to the nearest number so that some columns show over $100 \%$ response. 
and "proxy equipoise" - when staff and patients have faith in the consultant's conviction, and are content to rely on that and do not feel able or willing to form their own assessment. The high number of responses that could not simply be coded as "yes/no" illustrated how complex and ambiguous many respondents found the whole topic of breast cancer.

Equipoise is premised on uncertainty and ignorance, and respondents were asked for their views on informing patients about clinical uncertainty. Among the health professionals, $15 \%$ thought that women generally accepted current uncertainty about the nature and treatment of breast cancer when they were informed; $25 \%$ thought that women preferred to be informed; $15 \%$ thought that women were too distressed and preferred not to know; the rest gave other replies including discussion about how much the women and the range of relevant information varied. Responses varied widely from the view that patients were more trusting and confident when fully informed about uncertainty, to examples of women who were horrified by this information. When asked for their own views on current uncertainty about the nature, prevention and treatment of breast cancer, $32 \%$ of health professionals said they were "quite concerned" and the rest were equally divided between being "not worried", "very concerned" or giving qualified replies.

Among the screened women, $14 \%$ ticked "most treatments have been tested" and $56 \%$ indicated their belief that current knowledge is too limited. Treated women were asked if they would want to be informed about current uncertainty if they were asked to take part in a trial; $68 \%$ said "yes", $4 \%$ said "no", and the others gave qualified replies.

All respondents were asked how they might react if they were in a position to be asked to join three kinds of breast cancer trials involving surgery, chemotherapy, and the preventative tamoxifen trial for women at above average risk of developing breast cancer. Although 53\% of the professionals said they would agree to join the tamoxifen trial, in all the other replies from all three groups the refusals far exceeded agreement. Some professionals who supported randomisation in principle said they did not have equipoise for the actual trials discussed, or that they personally preferred the limited certainty of partially informed choice to the total uncertainty and powerlessness of being randomised. A radio- therapist and a senior nurse, who strongly supported randomised research in their general replies, changed when asked about their personal preference. The radiotherapist said that as someone used to making decisions she would not accept being randomised. The nurse said that she would choose to have all available treatments. Some treated women said they could not go through chemotherapy unless they were convinced that it was worthwhile; others said they wanted to have all available treatments, formally evaluated or not. Ethical ways of including people who do not have equipoise in randomised trials have been proposed. ${ }^{11}$

Health professionals, screened and treated women were all asked for their views on their preferred methods of making or sharing decisions. The replies are summarised here into three categories, noted in other research ${ }^{12}{ }^{13}$ : the patient actively deciding, with or without the doctor's advice; patients and doctors sharing decisions, and the "passive" category when doctors decide for patients (see Table 2 ). In relation to equipoise, "active deciders" tended to have a clearly preferred treatment although it is possible for understanding of uncertainty and acceptance of equipoise to support their informed consent to be randomised. The sharers include those who are willing to consent to research, and the "passive" group includes those who trust a clinician's decision to involve them in research. Table 2 reports subjective responses to our research questions and can only offer a rough indication of actual preferences and decision-making. The responses may be affected by respondents' modesty, caution or uncertainty.

Whilst most screened women replied that they preferred "sharing", treated women were more likely to say that they wanted either an active or a passive role. They tended to report experiencing less consultation than they wanted. Almost half the treated women said they wanted an active part in research decisions: professionals were more likely to believe that women wanted a collaborative, and not an active, role. Discussions about "sharing" ranged from wishing to take a minor to a major part in decisionmaking. Discussions about the complications of actual experiences, and people's reasoning and qualifications to their replies contribute the most important data, and are given in the full research report. $^{8}$

Acceptance of equipoise could be affected by personal belief in the importance of clinical research.

Table 2 Summary of views of all three groups on making and sharing decisions about treatment and research

\begin{tabular}{|c|c|c|c|c|}
\hline$\%$ Replies & Active & Sharing & Passive & Other response \\
\hline Screened women on preferred treatment decision method & 11 & 73 & 7 & 10 \\
\hline Treated women on preferred treatment decision method & \multirow{2}{*}{\multicolumn{2}{|c|}{$30^{\star}$}} & 20 & 30 \\
\hline Treated women on treatment decision method experienced & & & 26 & 44 \\
\hline Treated women on preferred research decision method & 47 & 21 & 24 & 9 \\
\hline Professionals on preferred research decision method & 29 & 38 & 12 & 21 \\
\hline
\end{tabular}

^ In these interviews, women described how they discussed options with their doctor. 
When asked for their general views on breast cancer research, the treated women expressed rather more confidence than the professionals did in the benefits of research (reduces mortality and morbidity, increases knowledge, offers hope). The professionals were more concerned about problems of research (of "using" people, reducing choice, increasing anxiety) than the treated women were. However, professionals were more likely $(83 \%)$ than the treated women $(80 \%)$ and the screened women $(22 \%)$ to say that trials are valuable in showing which treatments are harmful or useless.

Equipoise could also be affected by personal motives for or against supporting research, and a selection of replies on motives is given here. Some of the questions were slightly different for the three groups of respondents. One main motive for taking part in research, "to help other people", was given by $92 \%$ of treated women and $82 \%$ of professionals; $50 \%$ of screened women gave "to help to save other people's lives" as a motive, and $63 \%$ "to improve treatments in the future". The other main motive was "to help to find the answers", supported by $80 \%$ of treated women and $92 \%$ of professionals; $54 \%$ of screened women agreed with the motive "to help to increase medical knowledge". A third motive, "to increase my own chances of getting good care", was supported by $67 \%$ of professionals, $43 \%$ of treated women and $40 \%$ of screened women.

The following motives were given for refusing to take part in research: "Could not accept being randomised" - $58 \%$ of treated women, $56 \%$ of screened women, $39 \%$ of professionals; "If I objected to the trial design" $-85 \%$ of treated women, $97 \%$ of professionals; "Dislike lack of choice" - $71 \%$ of treated women; "Would want to share in choosing my treatment" - $56 \%$ of screened women. Treated women emphasised their desire for personal choice in contrast to impersonal chance. "I've done research myself, I know how important it is, but at that moment when I knew I had breast cancer I needed him [consultant] to know what was the best decision for me".

\section{Conclusion}

The pilot study raises questions which we intended to follow up in a larger project. We are cautious about generalising from the initial findings. However, the responses from a range of centres and from the 90 lengthy interviews suggest widespread ignorance and unease about equipoise, which cast doubt on the status of equipoise as the linchpin of ethical breast cancer trials. Equipoise may be more widely accepted in research about other diseases, with lower mortality and morbidity rates, less disparity between treatment arms, and shorter treatment episodes. The collective uncertainty which can justify equipoise did not seem to be shared by many of our respondents, only $23 \%$ of health professionals said that they were "very concerned" about the limits of current medical knowledge. This reported level of concern did not fitê? with the rising mortality rates for breast cancer in the $\Rightarrow$ UK and the seriously inadequate knowledge about $\stackrel{5}{+}$ the origins, prevention and treatment of the disease, ${ }^{14} \mathrm{C}$ reported in the professional ${ }^{15}$ and the popular press. ${ }^{16} \frac{\bar{O}}{\overline{0}}$ Until health professionals and the public are more aware and concerned about current uncertainty and@ ignorance, relatively few people will have personales equipoise. Communal equipoise will rest on the views $\vec{\circ}$ of a minority of experts rather than on a majority view, and a consensus of equipoise among members $\vec{\omega}$ of the research teams will be harder to achieve.

Nurses discuss trials with many women who are considering whether to enter them; nurses, radio-i graphers and other staff support women during the ${ }_{\omega}^{N}$ trials. The degree of equipoise shared by the breast cancer team, their commitment, even their unspokenor reservations, can affect women's decisions to enter? and to continue in trials.

Our research work included writing and evaluat $-\bar{C}$ ing a booklet for women who are asked to take part in breast cancer trials. The booklet explains research $\vec{c}$ terms and concepts, such as clinical uncertainty ando equipoise, which underlie informed consent to research. It suggests questions for women to use ifo they wish to discuss research with the breast cares team, to help professionals and patients to share⿳亠口冋 clear information. This exchange of views cano increase mutual understanding, and could help towards planning more widely acceptable trials $\overrightarrow{\overrightarrow{0}}$ which would meet high professional and public stan- 3 dards of equipoise. Attempts to interest the cancer information agencies in publicising the booklet, or $\mathrm{a}$. similar version, have failed. There appears to be much reluctance to inform the public about the uncertainty that underlies equipoise, and the mass? media tend to report the successes and hopes of cancer researchers rather than the extent of current? ignorance and uncertainty.

Replies in the breast cancer study to the questions on uncertainty and sharing decision 8 suggest that patients fall into three groups: those who wish to share in making decisions with their doctors, those who prefer their doctors to decide for them, and those who wish to be the "main decider" N about proposed treatment. In the breast cancer study, women were more concerned to be involved in decisions about research than about treatment although clinical research usually overlaps with treatment. In order to become informed and involved, patients have to be honestly told about uncertainty and risk. Some clinicians find this infor $\vec{D}$ mation too complex and distressing to share with their patients, and some argue that respect for informed consent can put too much constraint orर clinical trials. ${ }^{17}$ Our interviews revealed that some health professionals give over-optimistic estimations of success rates of treatment, and underestimate the urgent need for research. 
A partial solution to the conflict between informing patients honestly yet protecting them from extreme anxiety can be for clinicians to work out with their patients how much they wish to be informed and involved in decision-making. It is important not to confuse poor communication and information-giving, and lack of support and time, with patients' seeming lack of interest or understanding. Efforts to share informed decision-making may demand more time than staff are able to give. There are resource implications, which include time to inform practitioners and to help them to accept the limitations of current knowledge, as well as time to help their patients to come to terms with these uncertainties. The degree to which patients wish to be involved in decisions about trials will be influenced by their position on the equipoise spectrum: whether they want to think through all the options and come to a committed personal decision, or accept communal equipoise as a kind of majority view, or wish to defer to their doctor's decision and accept professional equipoise by proxy.

\section{Acknowledgements}

I thank my research colleagues in the breast cancer study, Mary Madden, Professor Ann Oakley and Dr Ruth Wilkins, everyone who took part in the interviews and the survey, all those who helped with gaining access to respondents, the research steering group, and the Cancer Research Campaign for sponsorship.

Priscilla Alderson, $B A, P h D$, is Senior Research Officer at the Social Science Research Unit, Institute of Education, University of London.

\section{References}

1 Baum M. The ethics of clinical research. In: Byrne P, ed. Ethics and law in health care and research. London: King's Fund, 1990.

2 Freedman B. Equipoise and the ethics of clinical research. New England fournal of Medicine 1987; 317: 141-5.

3 Collins R, Doll R, Peto R. Ethics of clinical trials. In: Williams C, ed. Introducing new treatments for cancer. Chichester: Wiley, 1992: 49-66.

4 Stiller C. Survival of patients in clinical trials and at specialist centres. See reference 3: 132.

5 Silverman W. Human experimentation: a guided step into the unknown. Oxford: Oxford University Press, 1985.

6 Gifford F. Community-equipoise and the ethics of randomised clinical trails. Bioethics 1995; 9,2: 127-48.

7 Ackner G. The ethics of ignorance. Fournal of Medical Ethics 1995; 21: 56.

8 Alderson P, Madden M, Oakley A, Wilkins R. Women's views of breast cancer treatment and research: report of the pilot project. London: Social Science Research Unit, Institute of Education, 1994.

9 Chouillet A, Bell C, Hiscox L. Management of breast cancer in southern England. British Medical fournal 1994; 308: 168-71.

10 See reference 8, and Thomson L. Combining care with randomised research: questions for breast care nurses. In: Alderson P, ed. Breast cancer, randomised controlled trials and consent. London: Social Science Research Unit, Institute of Education, 1994: 24-35.

11 Parmer M. Randomization before consent: practical and ethical considerations. See reference 3: 189-202.

12 Alderson P. Children's consent to surgery. Buckingham: Open University Press, 1993.

13 Ellis R, Leventhal B. Information needs and decisionmaking preferences of children with cancer. Psychooncology 1993; 2,4: 277-84.

$14 \mathrm{McPherson} \mathrm{K}$. What do we know about breast cancer and what do we need to know? See reference 10: 3-23.

15 Baum M. New approaches for recruitment into RCTs. Lancet 1993; 341: 812-3.

16 Batt S. Patient no more: the politics of breast cancer. London: Scarlet Press, 1994.

17 Tobias J, Souhami S. Fully informed consent can be needlessly cruel. British Medical fournal 1993; 307: 1199-201.

\section{News and notes}

\section{Attempt to meet patient concerns on medical records}

The Patients Association is to press for the establishment of a national committee to address the concerns of patients about their health and medical records, including accuracy, access and confidentiality, taking into account developments in electronic technology.

The proposer of the motion at PA's AGM, Rosamund Rhodes-Kemp, said that although patients could legitimately talk about "our records" they had no control over them. They now had the right of access to their own records, but what they were often given could be either some of them or nothing at all.

The computerising of records raised concerns about confidentiality, she said. Also patients needed to have access to their own records and they needed to stop others having access to them for commercial reasons. 